\title{
Acute Ocular Effects of Sphenopalatine Ganglion Nerve Block
}

\author{
Diane T Siegel ${ }^{1}$, Monica K Ertel ${ }^{2}$, Jennifer L Patnaik ${ }^{3}$, Nida S Awadallah ${ }^{4}$, Cara E Capitena Young ${ }^{5}$, Leonard K Seibold ${ }^{6}$,
} Malik Y Kahook

\begin{abstract}
Aim and objective: The goal of this study was to measure acute ocular effects in patients undergoing routine sphenopalatine ganglion (SPG) nerve block for headache. Projections from the SPG influence blood flow to the eye which may influence intraocular pressure (IOP). There are limited animal and human studies investigating the relationship between the SPG and its effect on the eye.

Materials and methods: This was a single-site, investigator-initiated, single-visit, prospective study. Participants were aged 18-85 years old who had consented to SPG nerve block for headache. The primary outcome measures were change in near visual acuity (NVA) and IOP preprocedure to immediately post-procedure. Additional data collected included pupil diameter and presence of any ocular or visual complaints. Results: A total of 13 patients were enrolled in the study. Average pre-procedure IOP was $14.2 \mathrm{~mm} \mathrm{Hg}$ [standard deviation (SD) 3.8] in the right eye and $13.7 \mathrm{~mm} \mathrm{Hg}$ (SD 3.2) in the left eye. Average post-procedure IOP was $14.8 \mathrm{~mm} \mathrm{Hg}$ (SD 3.8) in the right eye and $14.2 \mathrm{~mm} \mathrm{Hg}$ (SD 2.9) in the left eye. Neither the right nor left eye experienced a statistically significant change in IOP after SPG block. There were no statistically significant changes in average NVA or pupil diameter in either eye. There were no adverse events.

Conclusion and clinical significance: This pilot study suggests no significant acute changes in IOP or other ocular parameters after SPG block for headache disorders and supports the fact that the procedure is safe as it relates to ocular health. The ocular effects of SPG blockade merit further study in a larger cohort of patients.

Keywords: Headache, Intraocular pressure, Nerve block, Sphenopalatine ganglion.

Journal of Current Glaucoma Practice (2020): 10.5005/jp-journals-10078-1282
\end{abstract}

\section{INTRODUCTION}

The sphenopalatine ganglion (SPG), also called the pterygopalatine ganglion, is located in the pterygopalatine fossa just beneath the maxillary nerve and is the main parasympathetic ganglion of the upper jaw and related structures. ${ }^{1}$ It features complex anatomy and innervates blood vessels and glands of the nasal cavity, palate, lacrimal gland, eye, and cerebral circulation. ${ }^{1}$ Because of its anatomical connections and its role in the trigeminal-autonomic reflex, the SPG has been implicated in certain headache disorders and considered a therapeutic site for intervention. ${ }^{2}$ Sphenopalatine ganglion block is routinely performed by neurologists primarily for cluster headache and chronic migraine headache. ${ }^{3}$

Evidence suggests that projections from the SPG influence blood flow to the eye which can then influence intraocular pressure (IOP). ${ }^{4}$ Neurons from the SPG project to the ciliary body vasculature of the eye and contain nitric oxide synthase (NOS) and vasoactive intestinal peptide (VIP), which mediate vasodilation and increase blood flow. ${ }^{1}$ Activation of preganglionic input to the SPG via facial nerve stimulation has been shown to increase blood flow to the ciliary body; this effect can be blocked by a nicotinic acetylcholine receptor antagonist, hexamethonium. ${ }^{5}$

Animal models also show that NOS inhibition substantially reduces ( $\sim 50 \%$ ) ciliary blood flow. ${ }^{4}$ These animal models suggest a high parasympathetic resting tone that produces a tonic vasodilation of the ciliary body vasculature. Thus, decreasing parasympathetic innervation via this pathway may reduce blood flow and aqueous humor production and therefore lower IOP. ${ }^{4}$

There are limited animal and human studies investigating the relationship between parasympathetic input from the SPG and its effect on IOP. One study found long-lasting reduction in
1-3,5-7Department of Ophthalmology, Sue Anschutz-Rodgers Eye Center, The University of Colorado School of Medicine, Aurora, Colorado, USA

${ }^{4}$ Department of Family Medicine, The University of Colorado School of Medicine, Aurora, Colorado, USA

Corresponding Author: Malik Y Kahook, Department of Ophthalmology, Sue Anschutz-Rodgers Eye Center, The University of Colorado School of Medicine, Aurora, Colorado, USA, Phone: +1 720848-2020, e-mail: malik.kahook@cuanschutz.edu

How to cite this article: Siegel DT, Ertel MK, Patnaik JL, et al. Acute Ocular Effects of Sphenopalatine Ganglion Nerve Block. J Curr Glaucoma Pract 2020;14(2):57-60.

Source of support: Nil

Conflict of interest: None

IOP following removal of the SPG in monkeys. ${ }^{6}$ Another showed a moderate increase in IOP with stimulation of the SPG in monkeys. ${ }^{5}$ It is notable that the changes in IOP attributed to modulating blood flow to the ciliary body occur in a matter of minutes post-induction of treatment. Specifically, Nilsson et al. found an immediate increase in IOP with a steady-state level within 2 minutes. ${ }^{5}$ In the early 20th century, there was a report of modest IOP reduction after autonomic neurectomy of the sphenopalatine ganglia in patients with glaucoma. ${ }^{7}$ However, to the best of our knowledge, no English language studies in recent years have been performed to measure ocular effects of anesthetizing the SPG. ${ }^{8,9}$

This study aimed to measure the presence or absence of acute ocular effects in patients undergoing routine SPG nerve block for headache. We hypothesize that blood flow to the eye may be influenced by this procedure which, in turn, can influence IOP.

(-) The Author(s). 2020 Open Access This article is distributed under the terms of the Creative Commons Attribution 4.0 International License (https://creativecommons. org/licenses/by-nc/4.0/), which permits unrestricted use, distribution, and non-commercial reproduction in any medium, provided you give appropriate credit to the original author(s) and the source, provide a link to the Creative Commons license, and indicate if changes were made. The Creative Commons Public Domain Dedication waiver (http://creativecommons.org/publicdomain/zero/1.0/) applies to the data made available in this article, unless otherwise stated. 


\section{Materials and Methods}

This was a single-site, investigator-initiated, single-visit, prospective study to assess therapy-related changes in patients undergoing SPG block. The study adhered to the tenets of the Declaration of Helsinki and Institutional Review Board/Ethics Committee approval was obtained through the Colorado Multiple Institutional Review Board (COMIRB). Patients with a confirmed diagnosis of cluster headache or chronic migraine were recruited at their standardof-care neurology exam visits. Inclusion criteria were individuals 18-85 years of age who underwent SPG nerve block for cluster headache or chronic migraine and had consented to the procedure. Exclusion criteria included: (1) Inability to complete the ocular examinations required for this study and (2) Multiple nerve blocks being performed at the same session.

The primary outcome measures were change in near visual acuity (NVA) and IOP pre-procedure to immediately post-procedure. Additional data collected included pupil diameter and presence of any ocular or visual complaints. Study measurements were obtained immediately prior to the SPG block procedure and 15 minutes after the procedures were performed as outlined below:

- Near visual acuity: Near visual acuity was measured monocularly using the patient's current spectacle correction. A standard Rosenbaum Pocket Vision Screener card was held 14 inches from the eye in standard room lighting. Near visual acuity was recorded in Snellen equivalent for the smallest line of letters where the majority of numbers were read correctly.

- Pupil diameter: Measurement was obtained using the Oasis Colvard Pupillometer (Glendora, California, USA). This measurement was obtained by the examiner looking through the eyepiece to visualize the iris and pupil of the patient. The device has a reticule which superimposes a millimeter ruler over the image of the iris and pupil allowing for easy measurement of the pupil diameter.

- Tonometry: Measurement was performed using an FDA approved rebound tonometer (Icare ic100, Icare USA, Raleigh, North Carolina, USA). The IOP is obtained by a small probe which instantaneously rebounds off the corneal surface 6 times per eye in rapid succession. Rebound tonometry is used routinely in everyday ophthalmological examinations for IOP measurement.

- Assessment of visual and ocular complaints: Patients were asked to spontaneously report any observations pertaining to their vision as well as any general observations on ocular comfort. Responses were recorded verbatim.

Sphenopalatine ganglion blocks were planned for bilateral treatment according to standard of care. The procedure was performed under local anesthetic by dosing each nostril with $0.5 \mathrm{~mL}$ of $2 \%$ preservative-free lidocaine gel. Then, a lubricated spheno-catheter was placed in the nostril, parallel to the septum and above the middle turbinate, and advanced toward the apex of the nose approximately $5 \mathrm{~cm}$ posteriorly. Once resistance was felt, the catheter tip was deployed, delivering approximately $1.5 \mathrm{~mL}$ of 1 or $2 \%$ preservative-free lidocaine.

Following administration of the anesthetic, patients were maintained in a supine position for approximately 5 minutes and then repositioned upright. After 10 minutes in the seated position, the ocular measurements were repeated. Following the postprocedure measurements, the patients were managed by their neurology provider according to standard of care.
Results of the study were summarized using a combination of simple statistics [mean, standard deviation (SD), median, and range]. Paired $t$-tests were used to examine changes in ocular parameters before and after treatment for right and left eyes separately. The signed-rank test was also used to assess change before and after treatment for logMAR since it was not normally distributed. In addition, all right and left eyes were combined and analyzed using linear modeling with generalized estimating equations with a working unstructured correlation structure. These models account for the correlation of patients having two eyes and two time points included in the analysis. A $p$ value $<0.05$ was considered statistically significant.

\section{Results}

A total of 13 patients were enrolled in the study. Ten patients were Caucasian (non-Hispanic/Latino), two patients were Hispanic/ Latino, and one patient was Native American (Table 1). Twelve patients were female, and one patient was male. The age range was 23-71 years of age with a median of 32.5 years. Two patients had a history of photorefractive keratectomy previously. One patient had a history of non-exudative age-related macular degeneration. None of the patients had history of glaucoma, ocular hypertension, or ocular surgery within 6 months of testing and none were on IOP lowering medications. Twelve patients underwent bilateral SPG block. One patient underwent SPG block only on the right side due to a deviated septum preventing treatment on the contralateral side. This eye was excluded from ocular measurement data.

Ocular measures are presented in Table 2. Average NVA in the right eye prior to the SPG block was logMAR 0.07 (SD 0.15). After the procedure, the average NVA was logMAR 0.11 (SD 0.22). Average decline in vision for right eyes was logMAR 0.04 (SD 0.15), which was not significant (paired $t$-test $p=0.41$ ). Mean NVA in the left eye prior to the SPG block was logMAR 0.04 (SD 0.09) and these summary measures remained exactly the same for left eyes after the procedure; in fact, there was absolutely no individual change in vision for the 12 left eyes in the study. $p$ values for signed-rank test were also not significant ( $p=1.0$ for each eye). When all eyes were combined into one model, the change in logMAR remained not significant $(p=0.45)$.

Average pupil diameter prior to the procedure in the right and left eye was $4.8 \mathrm{~mm}$ (SD 1.1) and 4.9 (SD 1.0), respectively. After the procedure, the mean pupil diameter was $4.7 \mathrm{~mm}$ (SD 1.1) and 4.8 (SD 1.0) in the right and left eyes, respectively. Average change in

\begin{tabular}{ll}
\multicolumn{2}{l}{ Table 1: Participant characteristics } \\
\hline $\begin{array}{l}\text { Age, median } \\
\text { Sex }\end{array}$ & 32.5 years (range 23-71) \\
- Female & $12(92.3 \%)$ \\
Race & $1(7.7 \%)$ \\
- Caucasian, not Hispanic & $10(76.9 \%)$ \\
- Hispanic & $2(15.4 \%)$ \\
- Native American & $1(7.7 \%)$ \\
$\begin{array}{l}\text { Ocular history } \\
\text { - Photorefractive keratectomy }\end{array}$ & $2(15.4 \%)$ \\
- Non-exudative macular & $1(7.7 \%)$ \\
$\quad$ degeneration & \\
\hline
\end{tabular}


Table 2: Study results of ocular measures pre- and post-procedure

\begin{tabular}{|c|c|c|c|c|}
\hline & $\begin{array}{l}\text { Pre-procedure, } \\
\text { Mean (SD) }\end{array}$ & $\begin{array}{l}\text { Post-procedure, } \\
\text { Mean (SD) }\end{array}$ & $\begin{array}{l}\text { Change, } \\
\text { Mean (SD) }\end{array}$ & pvalue \\
\hline \multicolumn{5}{|c|}{ Near visual acuity Logarithm of the Minimum Angle of Resolution } \\
\hline Right eye & $0.07(0.15)$ & $0.11(0.22)$ & $0.04(0.15)$ & $0.41^{*}$ \\
\hline Left eye & $0.04(0.09)$ & $0.04(0.09)$ & $0(0)$ & $1.0^{*}$ \\
\hline All eyes & 0.06 & 0.07 & 0.02 & $0.45^{* *}$ \\
\hline \multicolumn{5}{|c|}{ Pupil size (mm) } \\
\hline Right eye & $4.8(1.1)$ & $4.7(1.1)$ & $-0.1(0.3)$ & $0.19 *$ \\
\hline Left eye & $4.9(1.0)$ & $4.8(1.0)$ & $-0.1(0.3)$ & $0.19 *$ \\
\hline All eyes & 4.8 & 4.7 & -0.1 & $0.15^{* *}$ \\
\hline \multicolumn{5}{|c|}{ Intraocular pressure $(\mathrm{mm} \mathrm{Hg})$} \\
\hline Right eye & $14.2(3.8)$ & $14.8(3.8)$ & $0.5(1.2)$ & $0.14^{*}$ \\
\hline Left eye & $13.7(3.2)$ & $14.2(2.9)$ & $0.5(1.2)$ & $0.18^{*}$ \\
\hline All eyes & 14.0 & 14.5 & 0.5 & $0.75^{* *}$ \\
\hline
\end{tabular}

${ }^{*} p$ values obtained from paired $t$-test

** $p$ values obtained from linear modeling to account for correlation of patients having two eyes and two time points included in the study. Standard deviations not provided for combined eyes due to this correlation SD, Standard Deviation

pupil diameter was a decrease of $0.1 \mathrm{~mm}$ in size, which was not statistically significant ( $p=0.19$ for each eye and 0.15 for all eyes).

Average pre-procedure IOP was $14.2 \mathrm{~mm} \mathrm{Hg}$ (SD 3.8) in the right eye and $13.7 \mathrm{~mm} \mathrm{Hg}$ (SD 3.2) in the left eye. Average postprocedure IOP was $14.8 \mathrm{~mm} \mathrm{Hg}$ (SD 3.8) in the right eye and 14.2 $\mathrm{mm} \mathrm{Hg}$ (SD 2.9) in the left eye. Average IOP increased by $0.5 \mathrm{~mm} \mathrm{Hg}$ (SD 1.2) in both the right eye ( $p=0.14)$ and the left eye $(p=0.18)$, an increase of $3.5 \%$ and $3.6 \%$, respectively. Neither the right nor left eye experienced a statistically significant change in IOP after SPG block, and this finding remained when all eyes were combined into one statistical model $(p=0.75)$. In four patients, IOP remained the same in both eyes before and after bilateral SPG block. In five patients, there was an IOP increase in both eyes after bilateral SPG block, and the patient with only the right eye included in the study had a slight increase in IOP. In two patients, there was an IOP reduction in both eyes after bilateral SPG block. In one patient, the IOP of the left eye remained the same and the IOP of the right eye decreased.

One patient had symptoms of visual aura in the right eye more than the left and experienced decreased visual aura after the SPG block. All other patients denied visual or ocular complaints.

\section{Discussion}

The purpose of this pilot study was to evaluate the acute ocular effects of SPG block. Animal studies have suggested a relationship between SPG block and IOP, but there are limited data studying the ocular effects of SPG block in humans. Our study did not find any statistically significant change in NVA, IOP, or pupil size when assessed after SPG block.

It is possible that our results did not reach statistical significance within the timeframe of the study. In the present study, IOP was measured 15 minutes after the SPG block. The goal of the current study was to evaluate the acute ocular effects of SPG blockade at a time point that is known to effect ganglion function. Past studies have shown measurable changes in IOP within 2 minutes of acting on the facial nerve to induce blood flow changes to the eye. ${ }^{5}$ Additionally, SPG block to treat headache disorders is known to alleviate symptoms within minutes of treatment indicating a direct influence on the SPG within that time-frame. It is, however, possible that significant changes in IOP or other metrics may be seen at later time points after administration of the block. In fact, prior animal studies linking SPG blockade with IOP lowering effect suggest the peak IOP lowering effect occurs days to weeks after the intervention. ${ }^{6}$ Future studies should be directed to more longitudinal follow-up after the SPG block.

Most patients in the study denied any immediate ocular or visual complaints with the procedure. One patient described a decrease in visual aura symptoms after the block. To the best of our knowledge, the existing literature does not suggest that there are significant, permanent ocular adverse events reported with SPG blockade. ${ }^{2,10}$ However, one small study suggests that basal rate of tear secretion is reduced with SPG blockade, which may contribute to dry eye syndrome. ${ }^{11}$ Overall, SPG blockade appears to be a safe and well-tolerated procedure with low risk of ocular side effects. $^{2}$

The study is not without limitations. As this was a pilot study, our sample size may be too small to detect statistically significant differences in ocular measures after SPG block. Most ocular measures showed minimal to no change, although there was an average increase in IOP of $0.5 \mathrm{~mm} \mathrm{Hg}$ among our study patients, but this change is clinically insignificant. We did not assess the tear film in this study. Sphenopalatine ganglion blockade may influence the tear film and subsequently visual acuity, although no such changes in NVA were noted. Additionally, the majority of study participants were female and Caucasian. However, epidemiological data report that females disproportionately suffer from headache disorders and thus our sample may be fairly representative of the population undergoing SPG block. ${ }^{12,13}$

Future directions include repeating the study with a larger, more diverse study population and with follow-up time points beyond 15 minutes after the procedure. Studying the ocular effects of SPG blockade in a population of patients with a history of glaucoma or ocular hypertension, as well as headache, may be more likely to demonstrate a significant relationship between SPG block and IOP. Studies have demonstrated that migraine is significantly more common in patients with normal tension glaucoma than in control patients or patients with "high-pressure glaucoma". ${ }^{14}$ This relationship suggests a common vascular etiology for both migraine and normal tension glaucoma, worth investigating further to determine potential for common treatment pathways.

\section{Conclusion and Clinical Significance}

In conclusion, this pilot study indicates the absence of any significant acute changes in IOP or other ocular parameters after SPG block treatment for headache disorders. Importantly, no significant adverse events were reported, supporting the fact that SPG block is a safe procedure as it relates to ocular health. Given the role that the SPG is believed to play in regulating blood flow to the eye, the ocular effects of SPG blockade merit further study in a larger cohort of patients.

\section{Acknowledgments}

The study adhered to the tenets of the Declaration of Helsinki and Institutional Review Board/Ethics Committee approval was obtained through the Colorado Multiple Institutional Review Board (COMIRB). 


\section{References}

1. Gibbins I. Chapter 5 - peripheral autonomic pathways. Hum Nerv Syst 2012. 141-185.

2. Robbins MS, Robertson CE, Kaplan E, et al. The sphenopalatine ganglion: anatomy, pathophysiology, and therapeutic targeting in headache. Headache J Head Face Pain 2016;56(2):240-258. DOI: 10.1111/head.12729.

3. Mojica J, Mo B, Ng A. Sphenopalatine ganglion block in the management of chronic headaches. Curr Pain Headache Rep 2017;21(6):1-8. DOI: 10.1007/s11916-017-0626-8.

4. McDougal DH, Gamlin PD. Autonomic control of the eye. Compr Physiol 2015;5(1):439-473.

5. Nilsson SFE, Linder J, Bill A. Characteristics of uveal vasodilation produced by facial nerve stimulation in monkeys, cats and rabbits. Exp Eye Res 1985;40(6):841-852. DOI: 10.1016/0014-4835(85)90129-0.

6. Ruskell GL. An ocular parasympathetic nerve pathway of facial nerve origin and its influence on intraocular pressure. Exp Eye Res 1970;10(2):319-330. DOI: 10.1016/S0014-4835(70)80044-6.

7. Golding-Wood PH. The ocular effects of autonomic surgery. Proc $\mathrm{R}$ Soc Med 1964;57(6):494-497.
8. Schuurmans R, Strebel P. Die beeinflussung des augeninnendrucks durch koagulation des nervus vidianus. Klin Monbl Augenheilkd 1980;177(10):459-462. DOI: 10.1055/s-2008-1057669.

9. Biankina IN, Katin VI. Use of pterygopalatine ganglionic block in the treatment of malignant glaucoma. Vestn Oftalmol 2006;122(2):35-36.

10. Binfalah M, Alghawi E, Shosha E, et al. Sphenopalatine ganglion block for the treatment of acute migraine headache. Pain Res Treat 2018;2018:2516953. DOI: 10.1155/2018/2516953.

11. Slade SG, Linberg JV, Immediata AR. Control of lacrimal secretion after sphenopalatine ganglion block. Ophthal Plast Reconstr Surg 1986;2(2):65-70. DOI: 10.1097/00002341-198601050-00002.

12. Burch RC, Loder S, Loder $\mathrm{E}$, et al. The prevalence and burden of migraine and severe headache in the United States: updated statistics from government health surveillance studies. Headache 2015;55(1):21-34. DOI: 10.1111/head.12482.

13. Manzoni GC, Stovner LJ. Epidemiology of headache. Handb Clin Neurol 2010;97:3-22.

14. Cursiefen $C$, Wisse $M$, Cursiefen $S$, et al. Migraine and tension headache in high-pressure and normal-pressure glaucoma. Am J Ophthalmol 2000;129(1):102-104. DOI: 10.1016/S0002-9394(99) 00289-5. 